\title{
Platelet activity and aspirin efficacy after off-pump compared with on-pump coronary artery bypass surgery: Results from the prospective randomized trial PRAGUE 11-Coronary Artery Bypass and REactivity of Thrombocytes (CABARET)
}

\author{
Frantisek Bednar, MD, PhD, ${ }^{a}$ Pavel Osmancik, MD, PhD, ${ }^{\mathrm{b}}$ Tomas Vanek, MD, PhD, ${ }^{\mathrm{a}}$ Heidi Mocikova, MD, PhD, ${ }^{\mathrm{c}}$
} Martin Jares, MD, ${ }^{a}$ Zbynek Straka, MD, PhD, and Petr Widimsky, MD, PhD, FESC ${ }^{b}$

From the Cardiocenter, Departments of Cardiac Surgery ${ }^{\mathrm{a}}$ and Cardiology, ${ }^{\mathrm{b}}$ and the Department of Clinical Hematology, ${ }^{\mathrm{c}}$ 3rd Medical School, Charles University and University Hospital Kralovske Vinohrady, Prague, Czech Republic.

Supported by research grant no. 8526-3/ 2005 from the Internal Grant Agency of the Ministry of Health of the Czech Republic.

Received for publication Oct 20, 2007; revisions received Feb 13, 2008; accepted for publication March 7, 2008.

Address for reprints: Frantisek Bednar, MD, $\mathrm{PhD}$, Cardiocenter, Department of Cardiac Surgery, Na Homolce Hospital, Roentgenova 2, 15030 Praha 5, Czech Republic (E-mail: fandabednar@email.cz).

J Thorac Cardiovasc Surg 2008;136:105460

\section{$0022-5223 / \$ 34.00$}

Copyright $\odot 2008$ by The American Association for Thoracic Surgery

doi:10.1016/j.jtcvs.2008.03.052
Objectives: Changes in hemostasis after off-pump coronary artery bypass grafting are still being discussed. There is a lack of information about platelet activity and aspirin efficacy after coronary artery bypass grafting. The aim of this study was to assess and compare platelet activity and aspirin efficacy early and late after off-pump and on-pump coronary artery bypass grafting.

Methods: Eighty patients were enrolled in a prospective randomized study. Platelet activity was determined based on membrane expression of antigen CD62P (P-selectin) by means of flow cytometric analysis. Aspirin efficacy was assessed by using arachidonic acid-induced platelet aggregation. Blood samples were collected before the operation, immediately postoperatively, and on days 1,2, 5, and 30 .

Results: In the off-pump group expression of P-selectin was markedly increased in comparison with preoperative values, with a maximum difference observed on day $2(+53 \%, P=.02)$, and it was significantly higher compared with that seen in the on-pump group on days 2 and $5(+53 \%$ vs $+4 \%, P=.004$, and $+20 \%$ vs $-16 \%$, $P=.005)$. On day 30, P-selectin expression was similar both between the groups and in comparison with the preoperative values. Arachidonic acid-induced platelet aggregation was gradually decreasing until day 30 , but on day 2 , there was an unexpected increase in aggregation that was more expressed in the off-pump group.

Conclusions: The platelet activity is higher in the early postoperative period in offpump compared with on-pump coronary artery bypass grafting. The present aspirin strategy seems to be insufficient in the early postoperative period, irrespective of the surgical technique used.

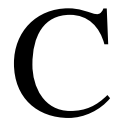
oronary artery bypass grafting $(\mathrm{CABG})$ performed with cardiopulmonary bypass (CPB) is associated with hemostatic changes that can either lead to early bleeding complications (postoperative bleeding) or, in contrast, to early thrombotic complications (generation of microthrombi and CPB-related organ dysfunction). These conditions are caused in various ways by changes in coagulation factors, fibrinolysis, and platelet dysfunction. ${ }^{1,2}$

Off-pump CABG surgery has been established as a less-invasive alternative to conventional CABG. Elimination of CPB leads to the reduction of hemostatic defects and consequently to less postoperative bleeding and fewer requirements for blood transfusion. ${ }^{3}$ However, several recent studies suggest that off-pump surgery could cause a procoagulant state, which appears within the first postoperative days and could possibly contribute to early thrombotic complications and affect early venous graft patency after off-pump CABG. ${ }^{4-7}$ Nevertheless, the described coagulation changes are often limited only to plasma markers, and not much is known about platelets 


\section{Abbreviations and Acronyms \\ $\mathrm{ACT}=$ activated clotting time \\ $\mathrm{CABG}=$ coronary artery bypass grafting \\ $\mathrm{CPB}=$ cardiopulmonary bypass \\ FITC = fluorescein isothiocyanate \\ MI = myocardial infarction \\ PRP = platelet-rich plasma}

\section{Heparin and Protamine Management}

In the on-pump group heparin was administered at an initial dose of $300 \mathrm{IU} / \mathrm{kg}$ to achieve an activated clotting time (ACT) of $480 \mathrm{sec}-$ onds. ACT was monitored every 30 minutes, and an additional heparin bolus (5000 IU) was administered when ACT was less than 440 seconds.

In the off-pump group heparin was administered at an initial dose of $150 \mathrm{IU} / \mathrm{kg}$ to achieve an ACT of more than 250 seconds. On completion of all anastomoses, the heparin effect was reversed with protamine chloride. An empiric dose regimen of $300 \mathrm{mg}$ (in the on-pump group) and $100 \mathrm{mg}$ (in the off-pump group) was administered, and if required, additional protamine was added to achieve an ACT of less than 130 seconds at the end of the operation.

and their activity in the first postoperative days after bypass surgery. ${ }^{8-11}$ The significance of platelets early after CABG is also supported by the fact that platelets play a key role in the process of arterial thrombosis and also the fact that the inhibition of platelets by early administered aspirin has a clear benefit against graft thrombosis and affects saphenous vein graft patency. ${ }^{12}$ In spite of this, changes in early and late platelet activity between off-pump and on-pump CABG and aspirin efficacy after both types of surgical intervention have not been thoroughly studied.

In the present prospective, randomized PRAGUE 11CABARET study, we evaluated the early and late postoperative platelet activity between on-pump and off-pump CABG and compared aspirin efficacy on inhibition of platelet aggregation early and late after both types of operation.

\section{Materials and Methods Patient Enrollment}

Eighty patients were randomized in a prospective study by using the envelope method ( $n=40$ for off-pump and $n=40$ for on-pump CABG surgery). Informed consent was obtained from all participants before enrollment in the study. The study protocol has been approved by the hospital ethics committee, and the study was performed in accordance with the Declaration of Helsinki. Patients were consecutively enrolled from May 2005 to December 2006. Inclusion criteria for enrollment of patients in this study were as follows: (1) indication for CABG surgery and (2) aspirin, heparin, or low-molecular-weight heparin withdrawal more than 7 days before the operation or clopidogrel withdrawal more than 14 days before the operation. Exclusion criteria were as follows: (1) nonstable angina pectoris, (2) acute myocardial infarction (MI) less than 30 days before the operation, (3) percutaneous coronary intervention less than 30 days before the operation, (4) stroke less than 6 months before the operation, (5) concomitant operation (valvular or MAZE procedure), (6) renal insufficiency (serum creatinine $>120 \mu \mathrm{mol} / \mathrm{L}$ ), (7) liver disorder, or (8) platelet count of less than 150,000 , prothrombin time greater than 1.2 international normalized ratio (INR), activated partial thromboplastin time test/control of greater than 1.2 , antithrombin III level of less than $80 \%$ or greater than $120 \%$, and fibrinogen level of less than 2 or greater than $6 \mathrm{~g} / \mathrm{L}$.

\section{Anesthetic and Surgical Technique}

Fast-track anesthesia and off-pump and on-pump operations were performed as described previously. ${ }^{13,14}$

\section{Perioperative and Postoperative Drug and Fluid Management}

In the perioperative period no antifibrinolytics (eg, aprotinin and tranexamic acid) were used in either group. Crystalloid solutions and $5 \%$ albumin solution were administered during the procedure. A red blood cell transfusion was administered when hemoglobin decreased to less than $8.5 \mathrm{~g} / \mathrm{dL}$ or hematocrit to less than $26 \%$. In the postoperative period a transfusion of fresh frozen plasma was administered when chest drain bleeding increased to more than $150 \mathrm{~mL} / \mathrm{h}$ or to more than $100 \mathrm{~mL} / \mathrm{h}$ for 2 consecutive hours. The red blood cell transfusion was administered according to similar criteria mentioned above. The platelet transfusion concentrates could be administered in the postoperative period when chest drain bleeding increased to $200 \mathrm{~mL} / \mathrm{h}$ for a consecutive 3 hours and platelet count was less than $60,000 / \mathrm{mm}^{3}$. No other prohemostatic agents were recommended according to the study protocol. Aspirin (200 $\mathrm{mg}$ ) was readministered 12 hours after the procedure in both groups. No additional antiaggregation or anticoagulation drugs (nonsteroidal anti-inflammatory drugs or low-molecular-weight heparin) were recommended during the first 5 days after the operation, according to the study protocol.

\section{Blood Sampling and Analyses}

Blood samples were collected in the morning before the operation, immediately after the operation (after skin closure, when the ACT was $<130$ seconds), and on postoperative days $1,2,5$, and 30 (days 1, 2, 5, and 30). At the same time points, the samples for routine coagulation tests and biochemical analyses were collected.

Platelet activity was determined by changes in the expression of the platelet surface antigen CD62P (P-selectin) by using flow cytometry, and aspirin efficacy was determined by means of light transmission aggregometry after the addition of arachidonic acid (AA). ${ }^{15}$ At the time points described above, $10 \mathrm{~mL}$ of venous blood was drawn in 2 citrated ( $9 \mathrm{NC}$ sodium citrate $3.2 \%$ ) vacutainers.

Flow cytometry. Platelet surface antigens were analyzed as described previously. ${ }^{16}$ The sample was stored for 2 hours at $37^{\circ} \mathrm{C}$. Supernatant was collected and platelet count was adjusted to $100 \times$ $10^{9} / \mathrm{L}$ with homologous plasma and phosphate-buffered saline. Fifty microliters of prepared sample was added to $50 \mu \mathrm{L}$ of the stabilizer Thrombofix (Immunotech, Beckman-Coulter Co). This mixture was incubated for 2 hours at $37^{\circ} \mathrm{C}$. Twenty microliters of prepared stabilized platelets were stained with the appropriate volume of

\section{Assays for Platelet Activity and Aspirin Efficacy}


mAbs at saturating concentrations: CD62P-fluorescein isothiocyanate (FITC)/CD41-phycoerythrin (Immunotech), CD62P-FITC/ CD42b (Immunotech), and isotype control IgG1-FITC/IgG1phycoerythrin (Immunotech). Stained samples were incubated for 10 minutes at room temperature, and $0.5 \mathrm{~mL}$ phosphate-buffered saline was added and mixed. The flow cytometer was calibrated with CYTO-COMP Reagent Kit (Immunotech) and the CYTOCOMP Cell Kit (Immunotech). Analysis was performed on an EPICS XL flow cytometer (Beckman-Coulter, Inc). The gating strategy of platelets was based on forward and side scatter and on the presence of CD42b antigen. At least 10,000 platelets were analyzed in each measurement. CD62P expression was determined as a percentage of positive cells.

Aggregometry. Platelet-rich plasma (PRP) was prepared immediately after blood collection by means of centrifugation of $5 \mathrm{~mL}$ of anticoagulated blood at $150 \mathrm{~g}$ for 10 minutes at room temperature. PRP was adjusted to a minimum platelet count of $150,000 / \mu \mathrm{L}$. Platelet-poor plasma was obtained from the remaining sample by means of centrifugation at $2500 \mathrm{~g}$ for 20 minutes. Samples were assayed on a PAP-4 Platelet Aggregation Profiler (BioBata Corp) with plateletpoor plasma as $100 \%$ of transmittance. The transmittance was set to $0 \%$ after adding $200 \mu \mathrm{L}$ of PRP. AA was added to each tube (the final concentration of AA in the PRP was $0.5 \mathrm{mg} / \mathrm{mL}$ ), and the aggregation profiles were plotted (percentage transmittance vs time). Platelet aggregability was calculated as total aggregation (percentage at 5 minutes). ${ }^{17}$

Test reliability. The intra-assay coefficient of variation was $3.2 \%$ $\pm 2.4 \%$ for AA aggregability and $3.8 \% \pm 4.1 \%$ for CD62P. The interassay (ie, intraindividual) variability was $8.9 \% \pm 10.2 \%$ for AA and $6.9 \% \pm 8.9 \%$ for CD62P in healthy non-aspirin-taking individuals.

\section{Power Calculation}

We were unaware of previous data comparing the difference in CD62P platelet membrane expression in patients after off-pump and on-pump CABG surgery. Thus we based our power calculations on the previous studies of comparing CD62P or other parameters of platelet activity in patients after percutaneous coronary intervention. We hypothesized a difference of two thirds of the standard deviation in logged data. Therefore we needed data from a minimum of $34 \mathrm{pa}-$ tients in each group for a $P$ value of less than .05 and a 1- $\beta$ value of greater than 0.8 .

\section{Statistical Analyses}

Continuous variables were compared by using a 2-sample $t$ test and categorical variables by using a $\chi^{2}$ test for independence in contingency tables. The time courses of AA, CD62P, and platelets counts were analyzed by means of analysis of variance for repeated measures. Correlations were evaluated by using the Spearman test. The tests are carried out at the 5\% significance level. The results are presented as means \pm standard deviation or medians with 95\% confidence intervals. Statistical analysis was performed with SPSS for Win v.14 (SPSS, Inc).

\section{Results}

\section{Patients' Characteristics}

Eighty patients were enrolled between May 2005 and December 2006, 40 in the off-pump and 40 in the on-pump group. Clinical variables of these patients are reported in Table 1. No significant differences between the groups were observed for demographics, preoperative risk factors, and medications. The basic preoperative hematologic parameters were also comparable (not shown). Perioperative and postoperative data are summarized in Table 2. Number of grafts, blood loss, heparin, and protamine doses were significantly higher in the on-pump group. The need for red blood cell and fresh frozen plasma transfusions was similar in both groups. In the on-pump group 2 patients received platelet transfusions postoperatively. In the off-pump group one patient received clopidogrel postoperatively. No significant differences in clinical outcome between groups were observed, and none of the patients died. In 4 patients ( 2 in the on-pump and 2 in the off-pump groups) there were signs of perioperative or postoperative MI. In 1 patient MI developed in the perioperative period, in 2 patients very early after admission to the intensive care unit and in 1 patient (on-pump group) after discharge from our department. There were no other signs of thrombotic complications.

\section{Platelet Activity and Aspirin Efficacy Studies}

Seven patients mentioned above were excluded from the analysis of platelet function (2 patients for platelet transfusion, 4 patients for $\mathrm{MI}$, and 1 patient for clopidogrel administration) because all these factors can affect the postoperative platelet functions.

The platelet counts before and after surgical intervention were similar and comparable in both groups (Figure 1). There was a trend toward a lower platelet count in the on-pump group (because of hemodilution), but there was no significant difference between groups. Platelet counts recovered to the preoperative values on day 5 and exceeded the preoperative values in both groups on day 30 .

Expression of P-selectin on the surface of platelets was comparable in both groups before the operation and on day 30 (Figure 2). In the off-pump group a significant increase

TABLE 1. Patients' characteristics

\begin{tabular}{lccc}
\hline & $\begin{array}{c}\text { On-pump } \\
\text { CABG (n = 40) }\end{array}$ & $\begin{array}{c}\text { Off-pump } \\
\text { CABG (n = 40) }\end{array}$ & $\begin{array}{c}\boldsymbol{P} \\
\text { value }\end{array}$ \\
\hline Age (y) & $64 \pm 11$ & $68 \pm 9$ & .11 \\
Male sex (\%) & 82 & 77 & .52 \\
Previous MI (\%) & 40 & 50 & .31 \\
Hypertension (\%) & 65 & 63 & .47 \\
Diabetes (\%) & 35 & 28 & .30 \\
Previous stroke (\%) & 3 & 5 & 1.0 \\
Hyperlipidemia (\%) & 67 & 55 & .73 \\
Smoking (\%) & 20 & 13 & .13 \\
Aspirin withdrawal (d) & $9 \pm 2$ & $9 \pm 4$ & .98 \\
\hline
\end{tabular}

Quantitative data are expressed as means \pm standard deviation. MI, Myocardial infarction. 
TABLE 2. Perioperative and postoperative data

\begin{tabular}{lccc}
\hline & $\begin{array}{c}\text { On-pump } \\
\text { group (n= 40) }\end{array}$ & $\begin{array}{c}\text { Off-pump } \\
\text { group (n = 40) }\end{array}$ & $\begin{array}{c}\boldsymbol{P} \\
\text { value }\end{array}$ \\
\hline No. of grafts & $2.4 \pm 0.6$ & $1.9 \pm 0.7$ & $<.01$ \\
CPB time (min) & $49 \pm 13$ & & \\
Crossclamp time (min) & $27 \pm 6$ & & \\
Heparin dose (IU $\times$ 1000) & $28.5 \pm 8.5$ & $8 \pm 5$ & $<.001$ \\
Protamine dose (mg) & $440 \pm 42$ & $150 \pm 65$ & $<.001$ \\
ACT (s) & & & \\
$\quad$ Before operation & $142 \pm 31$ & $139 \pm 36$ & .52 \\
$\quad$ End of operation & $128 \pm 8$ & $129 \pm 9$ & .67 \\
24-h Blood loss (mL) & $870 \pm 404$ & $660 \pm 364$ & .03 \\
FFP (\% of patients) & 35 & 28 & .30 \\
PRBC (\% of patients) & 43 & 37 & .28 \\
\hline
\end{tabular}

Quantitative data expressed as means \pm standard deviation. CPB, Cardiopulmonary bypass; IU, international unit; $A C T$, activated clotting time; $F F P$, fresh frozen plasma; $P R B C$, packed red blood cells.

of expression was recorded on day 2 in comparison with the preoperative values $(P=.02)$, and on days 2 and 5 , a significantly higher expression of P-selectin was recorded compared with that seen in the on-pump group $(P=.004$ on day 2 and $P=.005$ on day 5 ).

AA-induced platelet aggregation was similar in both groups before the operation (Figure 3). Immediately after the operation, platelet aggregation decreased significantly in both groups compared with the preoperative values, and only at that time was there a significant difference between the groups $(P=.04)$. On day 2 after the operation, despite the administration of aspirin, both groups showed an increase in platelet aggregation of greater than the values from day 1 ,

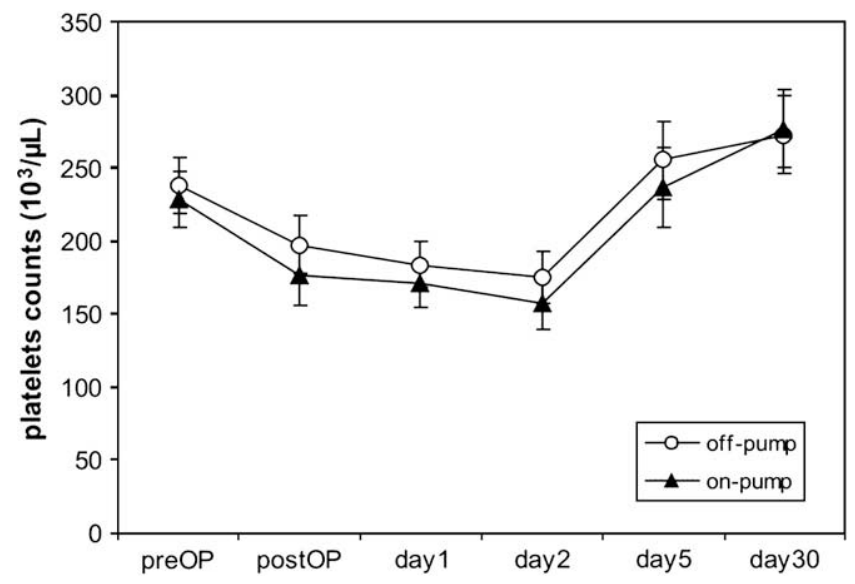

Figure 1. Platelet counts. Platelet counts in the off-pump and onpump groups at different time points. In the on-pump group there is a tendency toward lower platelet counts because of extracorporeal circulation. No statistically significant differences were found between the groups at any time points. Data are expressed as medians with $95 \%$ confidence intervals. preOP, Preoperative; postOP, postoperative. which was higher in the off-pump group. On day 5 after the operation, both groups showed a decrease in platelet aggregation in comparison with values from day 2 , and in the off-pump group the decrease in aggregation was much more significant. On day 30 after the operation, inhibition of platelet aggregation was almost the same in both groups and reached highly effective values.

Platelet activity and AA aggregability in 4 excluded patients with perioperative and postoperative $\mathrm{MI}$ are shown in Figure 4.

Finally, there was no statistically significant relationship between P-selectin expression and AA aggregability ( $R=0.15$ and $P=.34$ ). No significant relationship was noted between changes in 24-hour blood loss and in the preoperative, postoperative, and day 1 values of AA platelet aggregability $(R=0.10$ and 0.20 and $R=0.14)$ and similarly between 24-hour blood loss and P-selectin expression ( $R=0.24$ and 0.11 and $R=0.16$ ).

\section{Discussion}

Our study compared early and late postoperative platelet activity between the off-pump and on-pump CABG groups. Platelet activity, as determined by P-selectin expression, was significantly higher in the off-pump group on day 2 in comparison with the preoperative values and on days 2 and 5 and in comparison with values in the on-pump group. On day 30 , there was no difference between the groups and in comparison with preoperative values.

In the available literature there are few and conflicting data concerning changes in platelet activity after cardiac surgery. ${ }^{8-11,18-21}$ Therefore the interpretation of our results is not easy. The activation of platelets could be related only to the process of healing and to the changes in hemostasis, which are usually present in the early postoperative period. Higher platelet activity in off-pump surgery can be a "normal" state in the postoperative process of hemostasis restitution after a major operation. In contrast, this condition could be impaired in on-pump surgery due to hemostasis dysfunction caused by an extracorporeal circuit and other factors (eg, oxygenator, cardioplegia solution, hypothermia, thrombin, heparin, and protamine). However, some recent studies have indicated that the changes in hemostasis in terms of its increase (procoagulant state) could really exist after off-pump surgery. For example, Lo and colleagues ${ }^{4}$ and Quigley and associates ${ }^{5}$ describe that in off-pump surgery the response of the coagulation system (platelets were not studied) increased in the later period after the operation (in 20 to 96 hours or in 72 hours) in comparison with that after onpump surgery. It is not clear from the published studies whether platelets participate in this procoagulant state. Even though the role of platelets in this process has been questioned by some authors, with regard to the complexity of the hemostasis process, including interactions between the coagulation system of blood proteins and platelets, it is 

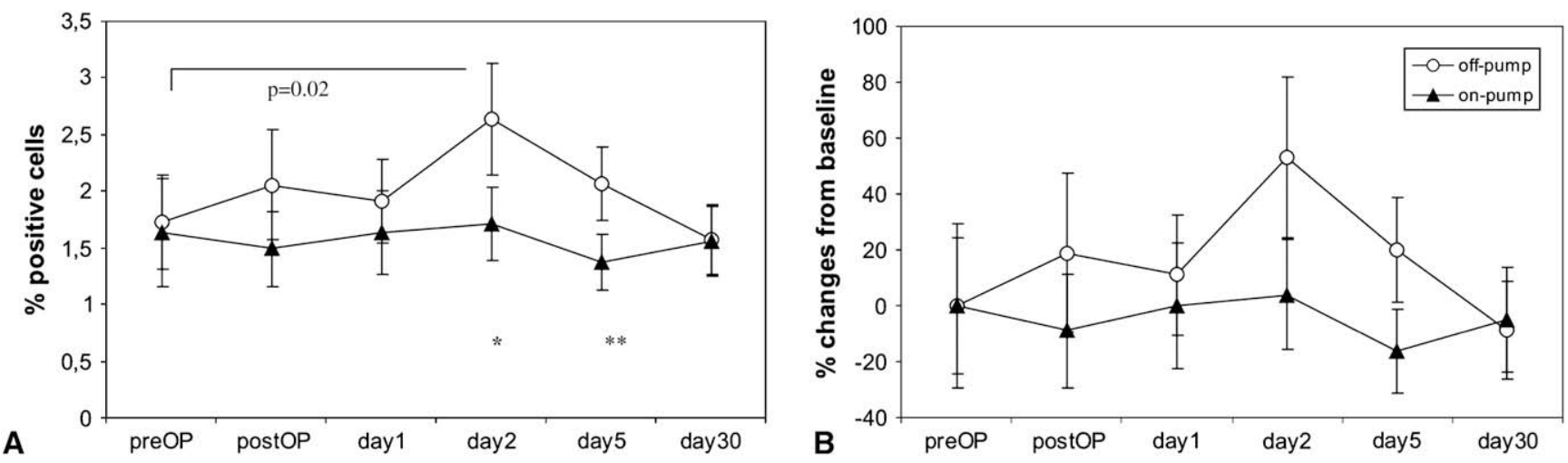

Figure 2. Platelet antigen CD62P (P-selectin) expression between the off-pump and on-pump groups. In the off-pump group expression of P-selectin significantly increased on day 2 in comparison with the preoperative values and remained significantly higher on days 2 and 5 in comparison with values in the on-pump group. Data are expressed as medians with $95 \%$ confidence intervals $(A)$ and as percentage change from baseline (B). preOP, Preoperative; post $O P$, postoperative. ${ }^{*} P=.004$ between off-pump and on-pump groups. ${ }^{*} P=.005$ between off-pump and on-pump groups.

very probable that platelets take part in this process. ${ }^{18}$ According to our results, platelets could play an important role in this state. Very few studies can be found in the available literature that would provide a comparison with our results by means of their methodology. A recently published study by Ballotta and coworkers ${ }^{8}$ also described changes in platelet activity (P-selectin and Annexin-V) but only very early after surgical intervention (2 hours). Similar to our study, it recorded an increase in P-selectin expression in both groups in the early postoperative period, but this increase was significantly higher in on-pump surgery compared with off-pump surgery. No comparisons in the later postoperative period were described. Parolari and associates ${ }^{20}$ did not find any significant changes in $\mathrm{P}$-selectin expression between off-pump and on-pump CABG on days 4, 8, and 30 after the operation, although they had a lower number of patients.
Higher platelet activity is associated with thrombotic complications in patients with coronary artery disease, and can be used to predict patients at risk of an adverse cardiovascular event (eg, reappearance of ischemia and the need for reintervention after percutaneous coronary intervention). ${ }^{22-24}$ It is not clear whether higher platelet activity can be associated with early bypass graft failure or whether it can contribute to negative clinical outcomes after bypass surgery. ${ }^{19} \mathrm{Al}-$ though it is not possible to make a valid conclusion from the platelet tests performed in our 4 patients with a postoperative MI (our study was not designed for this objective), at least 2 of them had significantly higher preoperative values of P-selectin. However, according to Poston and coworkers, ${ }^{18}$ what could cause graft failure is not the platelet activation itself but its interaction with coagulation cascade reactions, which appear on the surface of activated platelets
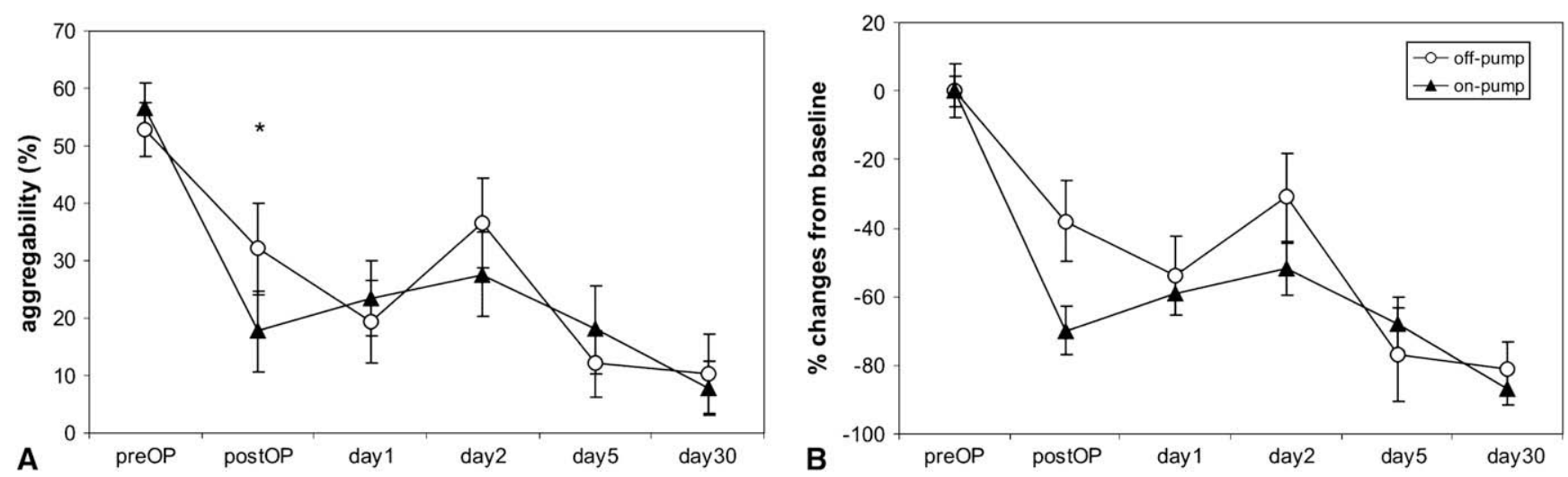

Figure 3. Arachidonic acid-induced platelet aggregation in the off-pump versus on-pump groups at different time points. Data are expressed as medians with $95 \%$ confidence intervals $(A)$ and as percentage change from baseline (B). preOP, Preoperative; postOP, postoperative. ${ }^{*} P=.04$ between off-pump and on-pump groups. 


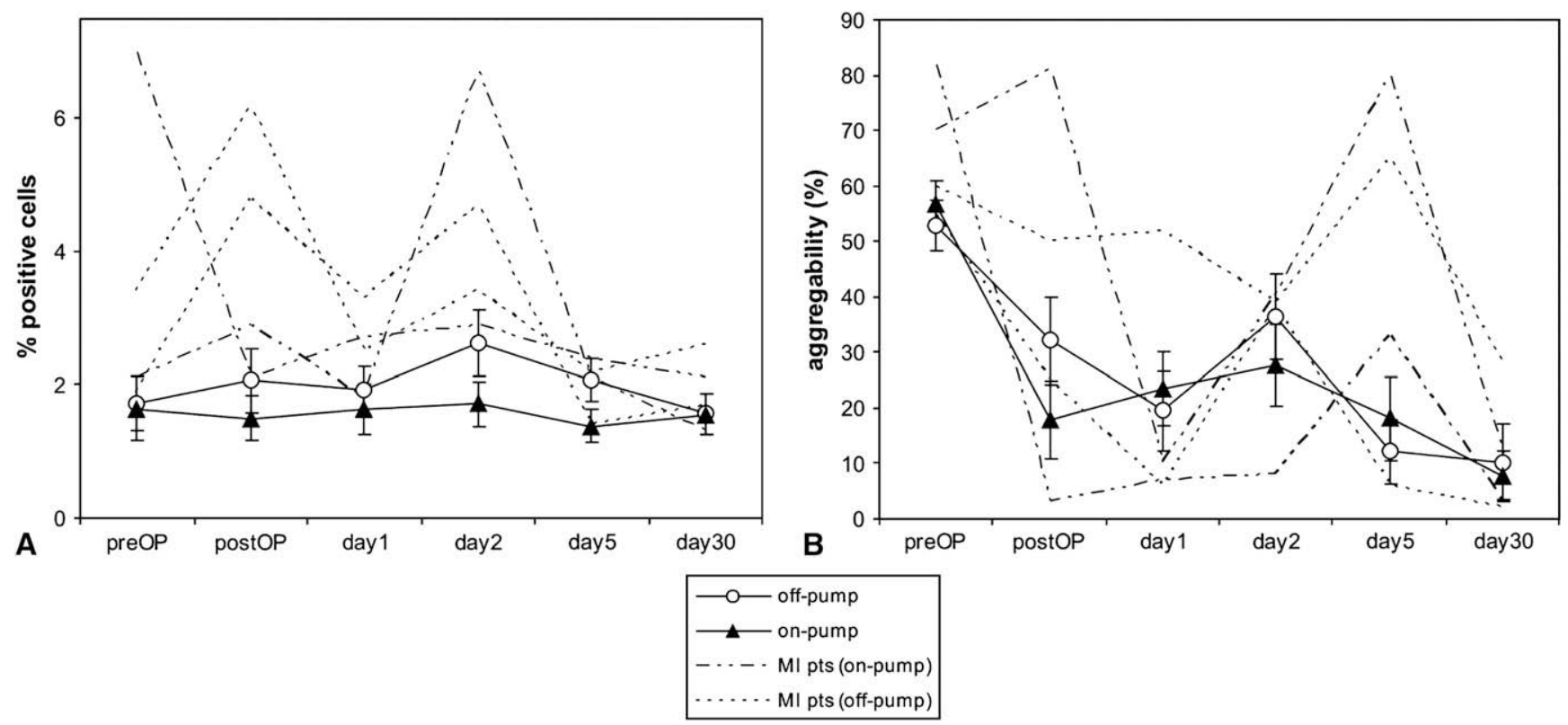

Figure 4. Platelet antigen CD62P (P-selectin) expression and arachidonic acid-induced platelet aggregation in 4 patients with perioperative or postoperative myocardial infarction. preOP, Preoperative; postOP, postoperative.

at the site of endothelial injury. Further studies are needed to clarify the complexity of these relations.

Apart from the assessment of platelet activity by means of P-selectin expression, we also focused on the evaluation of platelet aggregation after repeated administration of aspirin early after bypass surgery. It must be emphasized that P-selectin expression and platelet aggregation do not correlate together, which has been proved not only in our study but also in the available literature. ${ }^{25}$ Each of these factors describes something else in platelet function and activation. AA-induced platelet aggregation measured by means of light transmission aggregometry is today considered to be a reference method to evaluate aspirin efficacy, and its results provided very interesting findings. ${ }^{26}$ On postoperative day 2 , after previous reduction in aggregability caused by heparin and CPB (in the on-pump group), platelet aggregation increased unexpectedly and even more in the off-pump group. The increase in aggregability was recorded despite the administration of 2 doses of aspirin. Thus we can assume that platelet function is restored, but the inhibition effect of aspirin is still insufficient. This condition is more expressed in less dysfunctional platelets in the off-pump group. Unfortunately, changes in platelet aggregation were not measured on postoperative days 3 and 4 according to the study protocol. Therefore the expected inhibition effect of aspirin occurs approximately between postoperative days 3 and 5 . We can speculate whether the less dysfunctional platelets after off-pump surgery are, in contrast, more sensitive to the inhibition effect of aspirin in the later postoperative period, as the values of AA-induced platelet aggregation indicated on postoperative day 5 . Insufficient platelet inhibition early after bypass surgery was also described by Zimmermann and colleagues, ${ }^{27}$ who measured levels of thromboxane $\mathrm{B}_{2}$ in plasma after oral administration of aspirin. Similarly, Poston and coworkers ${ }^{19}$ recorded a significant increase in platelet resistance to aspirin effect in off-pump patients with early graft thrombosis. Aspirin insufficiency (resistance) early after bypass surgery is therefore a real problem to which more attention must be paid. The clinical effect of all these findings is also supported by the fact that the early postoperative period is extremely risky for early graft failure because of thrombosis. ${ }^{28}$ There arises a question whether modification or intensification of the dose regimen of aspirin or different antiplatelet strategies after off-pump and on-pump bypass surgery could lead to a more effective inhibition of aggregation with a minimum influence on postoperative bleeding and thus possibly to better clinical outcomes. Our results also support considerations about dual therapy with aspirin and clopidogrel, when clopidogrel, unlike aspirin, inhibits not only platelet aggregability but also P-selectin expression. ${ }^{29}$ However, to answer these and other questions, we need additional studies.

\section{Study Limitations}

Our trial includes a low number of patients, and it will be necessary to confirm or disprove our findings in future studies in a larger number of patients with a possible use of other sensitive markers of platelet activity and methods of measurement of aspirin efficacy. Our patients form a group of selected patients because our objective was to compare the influence of 2 different operative methods on the defined 
markers of platelet activity. The authors are aware of the possibility of obtaining different results in acute patients whose platelet activity might have already been primarily affected. Also, by excluding the patients with postoperative MI, the authors do not ignore the intention-to-treat rule, but they try to prevent the distortion of results by those patients who might primarily show higher postoperative platelet activity as a consequence of MI. Furthermore, we have to take into account different doses of heparin/protamine in both types of operation and their possible influence on platelet dysfunction. A certain limitation to the interpretation of the results is also caused by the fact that antifibrinolytics were not used. However, a distortion of the results could be caused by using a different dose in the off-pump and on-pump groups. Also, the administration of a 200-mg dose of aspirin does not correspond with standard guidelines, but this dose is usual in the Czech Republic because $325 \mathrm{mg}$ of aspirin is not registered in our country.

\section{Conclusions}

The PRAGUE 11-CABARET trial is the first prospective randomized study that demonstrates a significantly higher platelet activity in off-pump compared with on-pump CABG in the early postoperative period by means of a generally accepted marker of platelet activity. It also proves that aspirin insufficiency is a real problem that is present in the early postoperative period, and it is probably expressed in different ways between the off-pump and onpump CABG operations.

\section{References}

1. Khuri SF, Wolfe JA, Josa M, Axford TC, Szymanski I, Assousa S, et al. Hematologic changes during and after cardiopulmonary bypass and their relationship to the bleeding time and nonsurgical blood loss. J Thorac Cardiovasc Surg. 1992;104:94-107.

2. Paparella D, Brister SJ, Buchanan MR. Coagulation disorders of cardiopulmonary bypass: a review. Intensive Care Med. 2004;30: 1873-81.

3. Sellke FW, DiMaio JM, Caplan LR, Ferguson TB, Gardner TJ, Hiratzka LF, et al. Comparing on-pump and off-pump coronary artery bypass grafting: numerous studies but few conclusions: a scientific statement from the American Heart Association council on cardiovascular surgery and anesthesia in collaboration with the interdisciplinary working group on quality of care and outcomes research. Circulation. 2005; 111:2858-64.

4. Lo B, Fijnheer R, Castigliego D, Borst C, Kalkman CJ, Nierich AP. Activation of hemostasis after coronary artery bypass grafting with or without cardiopulmonary bypass. Anesth Analg. 2004;99:634-40.

5. Quigley RL, Fried DW, Salenger R, Pym J, Highbloom RY. Thrombelastographic changes in OPCAB surgical patients. Perfusion. 2002;17: 363-7.

6. Mariani MA, Gu YJ, Boonstra PW, Grandjean JG, van Oeveren W, Ebels T. Procoagulant activity after off-pump coronary operation: is the current anticoagulation adequate? Ann Thorac Surg. 1999;67: 1370-5.

7. Kurlansky PA. Is there a hypercoagulable state after off-pump coronary artery bypass surgery? What do we know and what can we do? J Thorac Cardiovasc Surg. 2003;126:7-10.
8. Ballotta A, Saleh HZ, El Baghdady HW, Gomaa M, Belloli F, Kandil H, et al. Comparison of early platelet activation in patients undergoing onpump versus off-pump coronary artery bypass surgery. $J$ Thorac Cardiovasc Surg. 2007;134:132-8.

9. Moller $\mathrm{CH}$, Steinbruchel DA. Platelet function after coronary artery bypass grafting: is there a procoagulant activity after off-pump compared with on-pump surgery? Scand Cardiovasc J. 2003;37:149-53.

10. Bidstrup BP, Scarrott $\mathrm{H}$, Luque M. Platelet function after off pump coronary surgery. Heart Surg Forum. 2003;6:286-7.

11. Gerrah R, Snir E, Brill A, Varon D. Platelet function changes as monitored by cone and plate(let) analyzer during beating heart surgery. Heart Surg Forum. 2004;7:E191-5.

12. Collaborative overview of randomised trials of antiplatelet therapy-II: maintenance of vascular graft or arterial patency by antiplatelet therapy. Antiplatelet Trialists' Collaboration. BMJ. 1994;308:159-68.

13. Vanek T, Brucek P, Straka Z. Fast track as a routine for open-heart surgery. Eur J Cardiothorac Surg. 2002;21:369-70.

14. Straka Z, Widimsky P, Jirasek K, Stros P, Votava J, Vanek T, et al. Offpump versus on-pump coronary surgery: final results from a prospective randomized study PRAGUE-4. Ann Thorac Surg. 2004;77:789-93.

15. Michelson AD. Platelet function testing in cardiovascular diseases. Circulation. 2004;110:e489-93.

16. Osmancik PP, Bednar F, Mocikova H, Stros P, Jirasek K, Straka Z, et al. The comparison of platelet activity between patients with patent vs. occluded coronary artery bypass grafts. Thromb Res. 2007;120:523-9.

17. Burke J, Kraft WK, Greenberg HE, Gleave M, Pitari GM, VanBuren S, et al. Relationship of arachidonic acid concentration to cyclooxygenasedependent human platelet aggregation. J Clin Pharmacol. 2003;43: 983-9.

18. Poston R, Gu J, Brown J, Gammie J, White C, Manchio J, et al. Hypercoagulability affecting early vein graft patency does not exist after off-pump coronary artery bypass. J Cardiothorac Vasc Anesth. 2005;19:11-8.

19. Poston R, Gu J, Manchio J, Lee A, Brown J, Gammie J, et al. Platelet function tests predict bleeding and thrombotic events after off-pump coronary bypass grafting. Eur J Cardiothorac Surg. 2005;27:584-91.

20. Parolari A, Mussoni L, Frigerio M, Naliato M, Alamanni F, Polvani GL, et al. The role of tissue factor and P-selectin in the procoagulant response that occurs in the first month after on-pump and off-pump coronary artery bypass grafting. J Thorac Cardiovasc Surg. 2005;130:1561-6.

21. Czerny M, Baumer H, Kilo J, Zuckermann A, Grubhofer G, Chevtchik $\mathrm{O}$, et al. Complete revascularization in coronary artery bypass grafting with and without cardiopulmonary bypass. Ann Thorac Surg. 2001;71:165-9.

22. Blann AD, Nadar SK, Lip GY. The adhesion molecule P-selectin and cardiovascular disease. Eur Heart J. 2003;24:2166-79.

23. Kabbani SS, Watkins MW, Ashikaga T, Terrien EF, Holoch PA, Sobel BE, et al. Platelet reactivity characterized prospectively: a determinant of outcome 90 days after percutaneous coronary intervention. Circulation. 2001;104:181-6.

24. Tschoepe D, Schultheiss HP, Kolarov P, Schwippert B, Dannehl K, Nieuwenhuis HK, et al. Platelet membrane activation markers are predictive for increased risk of acute ischemic events after PTCA. Circulation. 1993;88:37-42.

25. Li N, Hu H, Hjemdahl P. Aspirin treatment does not attenuate platelet or leukocyte activation as monitored by whole blood flow cytometry. Thromb Res. 2003;111:165-70.

26. Lordkipanidze M, Pharand C, Schampaert E, Turgeon J, Palisaitis DA, Diodati JG. A comparison of six major platelet function tests to determine the prevalence of aspirin resistance in patients with stable coronary artery disease. Eur Heart J. 2007;28:1702-8.

27. Zimmermann N, Wenk A, Kim U, Kienzle P, Weber AA, Gams E, et al. Functional and biochemical evaluation of platelet aspirin resistance after coronary artery bypass surgery. Circulation. 2003;108:542-7.

28. Motwani JG, Topol EJ. Aortocoronary saphenous vein graft disease: pathogenesis, predisposition, and prevention. Circulation. 1998;97:916-31.

29. Klinkhardt U, Bauersachs R, Adams J, Graff J, Lindhoff-Last E, Harder S. Clopidogrel but not aspirin reduces P-selectin expression and formation of platelet-leukocyte aggregates in patients with atherosclerotic vascular disease. Clin Pharmacol Ther. 2003;73: 232-41. 